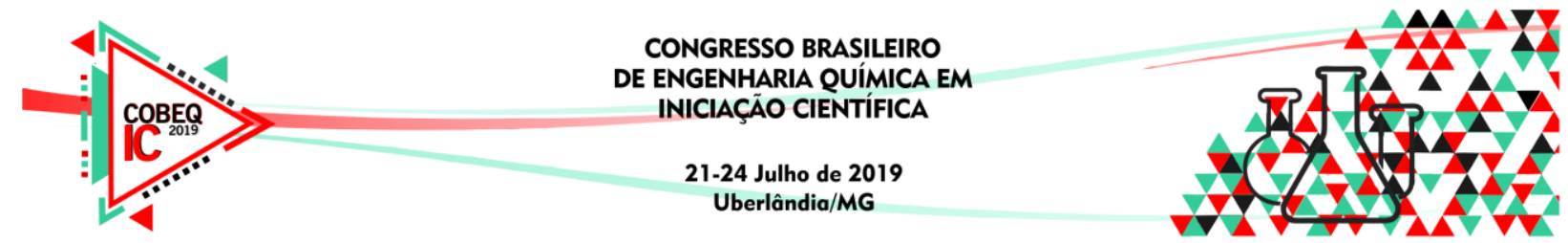

\title{
VÁlVULA DE CONTROLE DE BAIXO CUSTO PARA PROCESSOS QUÍMICOS E BIOQUÍMICOS
}

\author{
A. M. SILVA ${ }^{1}$ e A. VALDMAN ${ }^{2}$. \\ ${ }^{1}$ Universidade Federal do Rio de Janeiro, Escola de Química. \\ ${ }^{2}$ Universidade Federal do Rio de Janeiro, Departamento de Engenharia Química \\ E-mail para contato: alissonms@eq.ufrj.br
}

\begin{abstract}
RESUMO - No mercado de instrumentação, altos custos associados demandam a disponibilidade de alternativas de baixo preço de diversos instrumentos de processo para viabilizar o uso acadêmico. A plataforma Arduino surgiu em 2005, a partir da necessidade do barateamento e da simplificação na construção de circuitos eletroeletrônicos, aumentando o fator custo-benefício no desenvolvimento de plataformas de automação didáticas. A flexibilidade e fácil implementação da tecnologia apresentam-se como oportunidade de aplicação por usuários com conhecimento mínimo de eletroeletrônica. A partir da composição de placas semelhantes a microcomputadores e um software de código aberto para construção de algoritmos em linguagem de alto nível, o presente trabalho desenvolveu uma miniplanta composta por tanque que realiza recirculação de água utilizando uma bomba, com medidor de vazão e uma válvula manual do tipo gaveta acionada por motor de passo ligado a sua haste. A partir desta planta, foi desenvolvida uma curva de calibração, que permitiu a caracterização da válvula do tipo raiz quadrada.
\end{abstract}

\section{INTRODUÇÃO}

As válvulas são equipamentos destinados a estabelecer, controlar e/ou interromper o fluxo de fluidos em uma tubulação (SILVA, 2008). Elas podem ser classificadas quanto à forma de operação e o tipo de atividade que ela é preferencialmente destinada. Multifuncionalidade e o fator de na indústria os custos em tubulações representarem mais de metade do orçamento (TELLES, 2008) justificam estudos de desenvolvimento de alternativas de baixo custo para esses equipamentos.

A plataforma Arduino encontra-se bastante difundida como uma alternativa de baixo custo para monitorar variáveis em tempo real. Atualmente, encontra-se disponível no mercado uma ampla gama de sensores que medem variáveis de processo (temperatura, nível, pressão, vazão), permitindo a utilização destes recursos na divulgação da ciência e tecnologia como uma área de acesso a todos.

Em paralelo, com a era digital e a partir da popularização dos sistemas industriais de infraestrutura de dados, conceitos modernos como Big Data, Internet das Coisas (IOT) e Indústria 4.0 passaram a fazer parte do cotidiano de engenheiros de processo. 


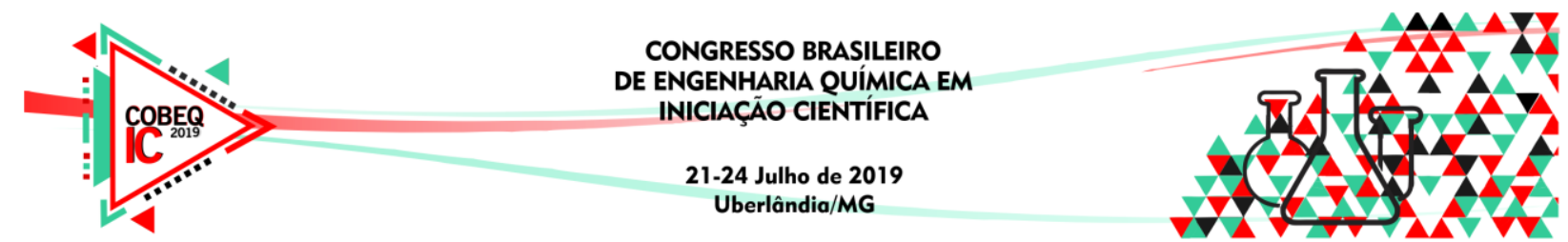

Para elaboração do trabalho, foi utilizado o software PI System ${ }^{\text {TM }}$ (OSISOFT, 2018), um sistema industrial para acompanhamento de dados de processo. Ele é capaz de estabelecer comunicação com servidores OPC, OLE for Process Control (em tradução livre, vinculação e incorporação de objetos para controle de processos), permitindo a interface de dados entre o software e a plataforma Arduino.

Agregando recursos de software industriais aos recursos de hardware de instrumentação de baixo custo, foi produzida uma miniplanta que permitiu a caracterização de uma válvula gaveta utilizando um transferidor e um motor de passo associado a sua haste e acionado via servidor OPC. O custo foi total de implementação foi de aproximadamente $\mathrm{R} \$ 150,00$, preço significativamente menor se comparado a uma válvula de controle industrial.

\section{DESENVOLVIMENTO DA PLANTA}

A miniplanta representada na Figura 1 trata-se de um sistema com recirculação composto por um tanque cilíndrico (volume: $12 \mathrm{~L}$ ), uma válvula gaveta (KLMX Metais, diâmetro: 1/2") e uma bomba centrífuga (Emicol Eletro Eletrônica, potência: 34 W). A instrumentação é composta por uma placa Arduino, um sensor de vazão (SEA Eletric, modelo: YF-S201B, faixa de medição 17 a $500 \mathrm{~mL} / \mathrm{s}$, precisão de 90\%), um módulo conversor eletrônico (Texas Instruments, modelo: ULN2003A) para acionar o motor de passo (Kiatronics, modelo: 28BYJ-48, faixa do torque: 6 a $1210^{-2}$.N.m), que está acoplado a haste da válvula, além de quatro botões para permitir testes de acionamento automático da válvula e ativação da bomba centrífuga.

Figura 1 - Representação da miniplanta

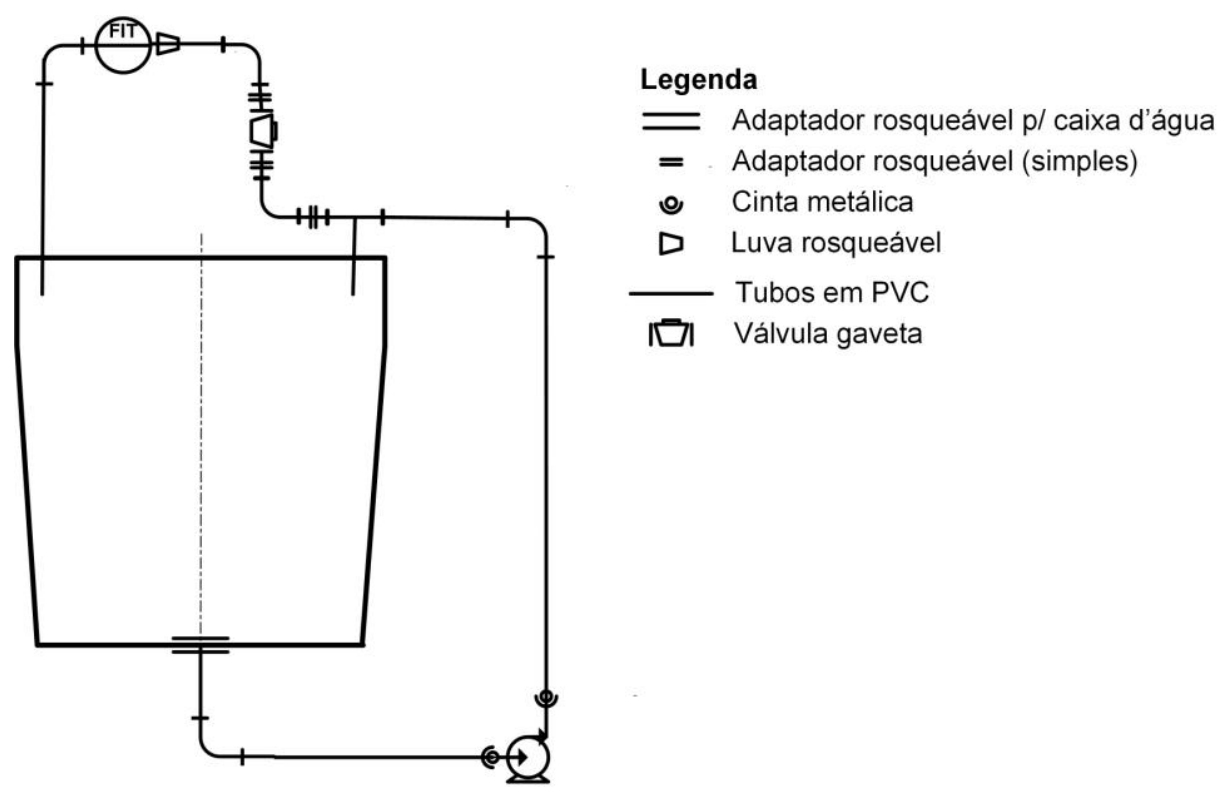

Um circuito elétrico foi desenvolvido para implementação da instrumentação previamente definida, conforme exibido na figura 2. Além disso, foi desenvolvido um código através da interface de desenvolvimento dedicada. O código consistiu em instruções para captura e envio em tempo real de informações das medidas de vazão e vazão acumulada. Ela também permitiu a conversão do percentual de abertura da válvula em tempo de 


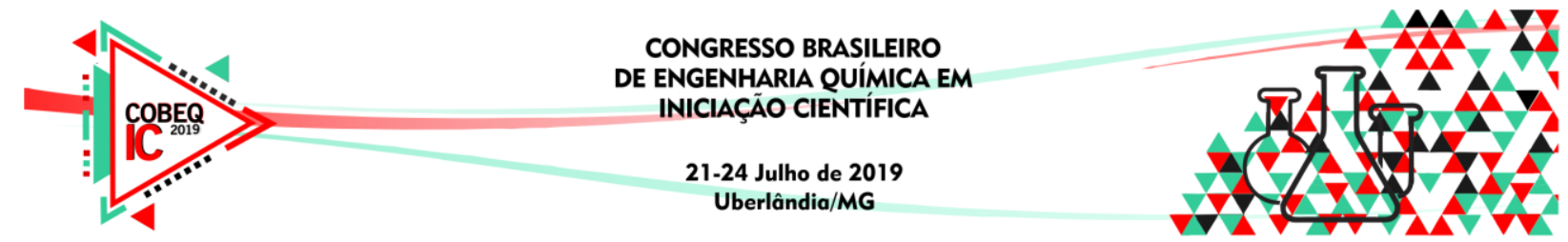

funcionamento do motor de passo e seu acionamento através de um servidor Arduino OPC. Vale ressaltar que foi utilizado ainda um relé de 2 canais para ligar e desligar a bomba e o motor de passo.

Figura 2 - Diagrama elétrico desenvolvido

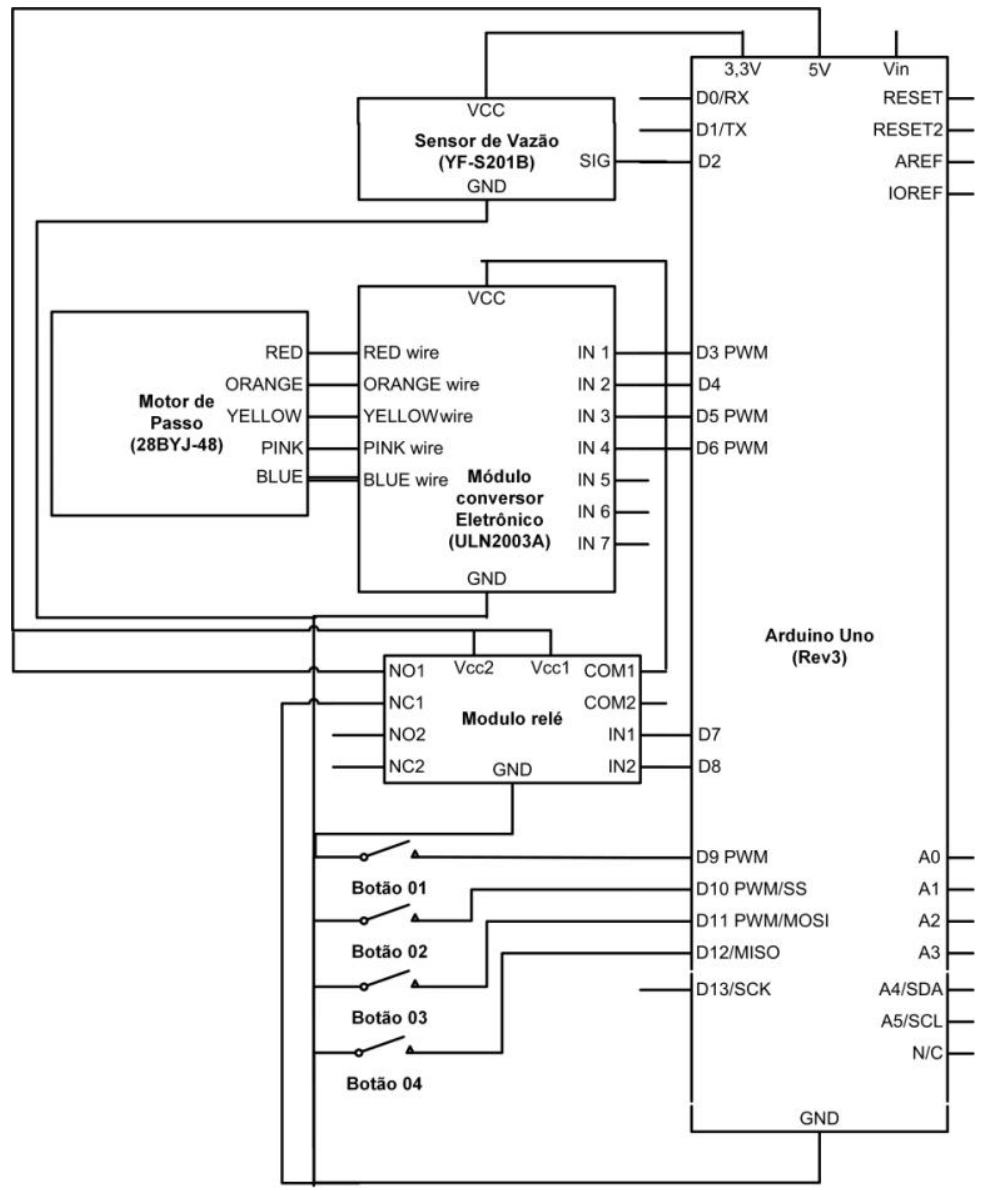

Além disso, no código desenvolvido, produziu-se um algoritmo que permite a identificação da posição atual do motor de passo e que possibilita aos botões abrir ou fechar a válvula completamente, bem como ligar e desligar o motor de passo e a bomba centrífuga.

\section{TESTES EXPERIMENTAIS}

Foi utilizado para acompanhamento e monitoramento dos dados o software PI System $^{\text {TM }}$. Foi configurada uma interface de comunicação entre o PI System e o servidor Arduino OPC, além do desenvolvimento de uma base de dados contendo as variáveis de processo de interesse para coleta e registro de valores. Para acompanhamento em tempo real dos dados foi construída uma interface gráfica exibida na figura 3. Através dela, é possível visualizar valores de vazão em tempo real, além de alternar a exibição dos gráficos de vazão e totalização de vazão. Pela interface, ainda se pode indicar valor percentual de abertura da válvula, acionar o motor de passo e a bomba centrífuga. $O$ modo de operação automático/manual e o valor do setpoint foram configurados para futuras implementações da malha fechada de controle. 


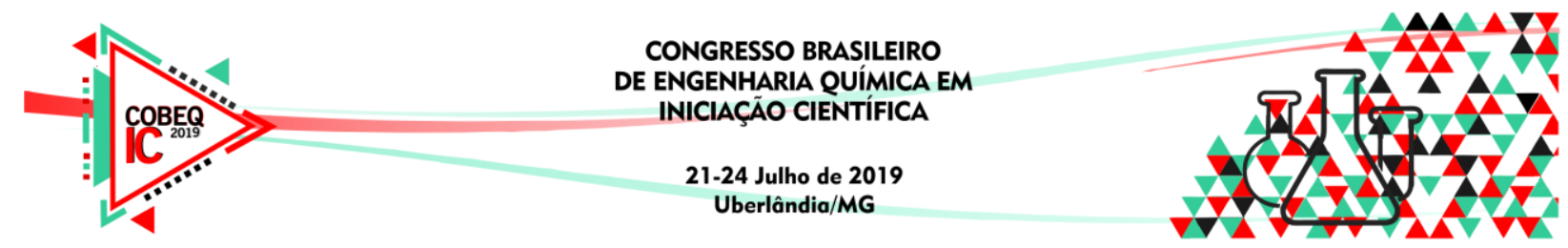

Figura 3 - Interface gráfica da planta
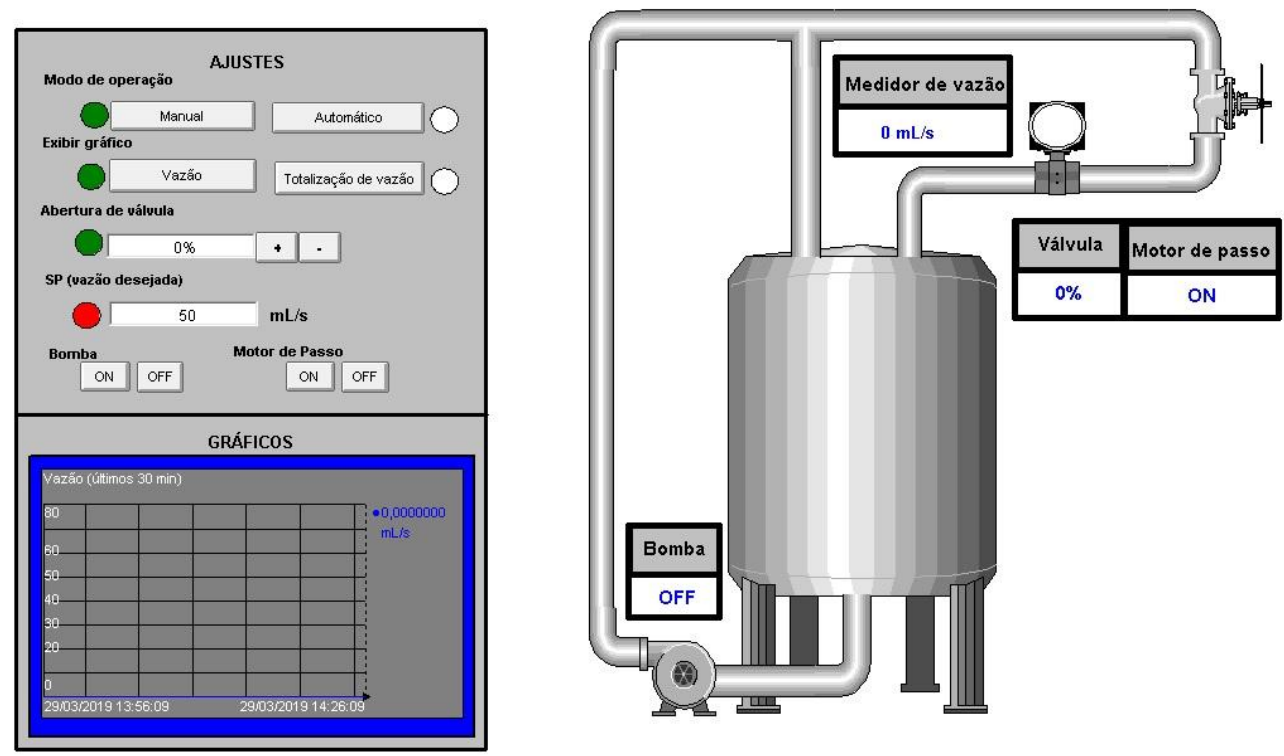

\section{RESULTADOS}

Através do registro dos valores de vazão, pode-se realizar a caracterização adequada do elemento de atuação da planta piloto. Para isso, produziu-se uma curva de calibração da válvula, baseado nos conceitos da norma ANSI/ISA-75.25.01 (ANSI, 2010).

Inicialmente, foi realizado um estudo de correlação entre percentual de abertura da válvula e ângulo de rotação do volante. Para isso, foi acoplado um transferidor para determinar o intervalo de abertura da válvula $\left(1580^{\circ}\right)$. Identificou-se que a cada $25 \%$ de abertura, o volante é girado $395^{\circ}$.

Com procedimento semelhante ao realizado por Zhu e Zhang (2017) e indicado por Baumann (2009), através de testes em triplicata, realizados tanto de forma ascendente quanto descendente, submeteu-se a válvula a diferentes taxas de abertura de intervalos consecutivos de $25 \%$ de abertura $(0 \%, 25 \%, 50 \%, 75 \%$ e $100 \%)$.

A figura 4 apresenta uma proposta de algoritmo desenvolvida para a realização automática destes testes. A cada abertura foi registrado o valor da vazão durante 1 minuto, e computada a média e o desvio padrão. Para esses cálculos foram descartados os oito primeiros e últimos valores, por apresentarem efeitos de oscilação sendo associados ao tempo de resposta da válvula. Por fim, foi calculada a média e desvio padrão das triplicatas por abertura. 


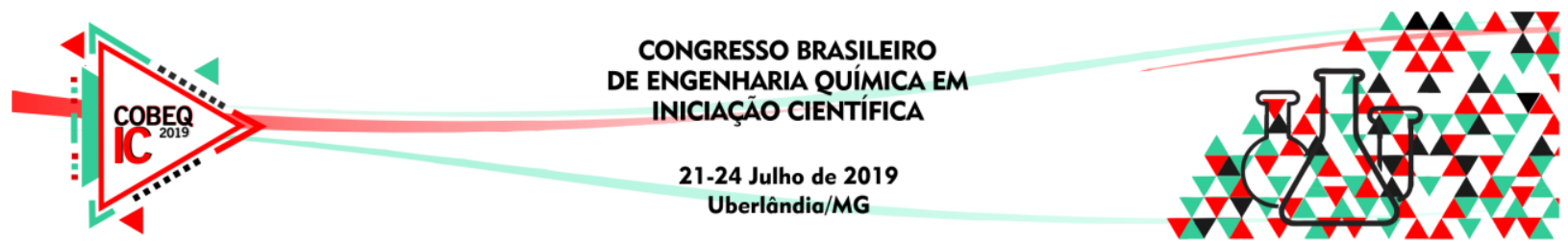

Figura 4 - Fluxograma da caracterização da válvula

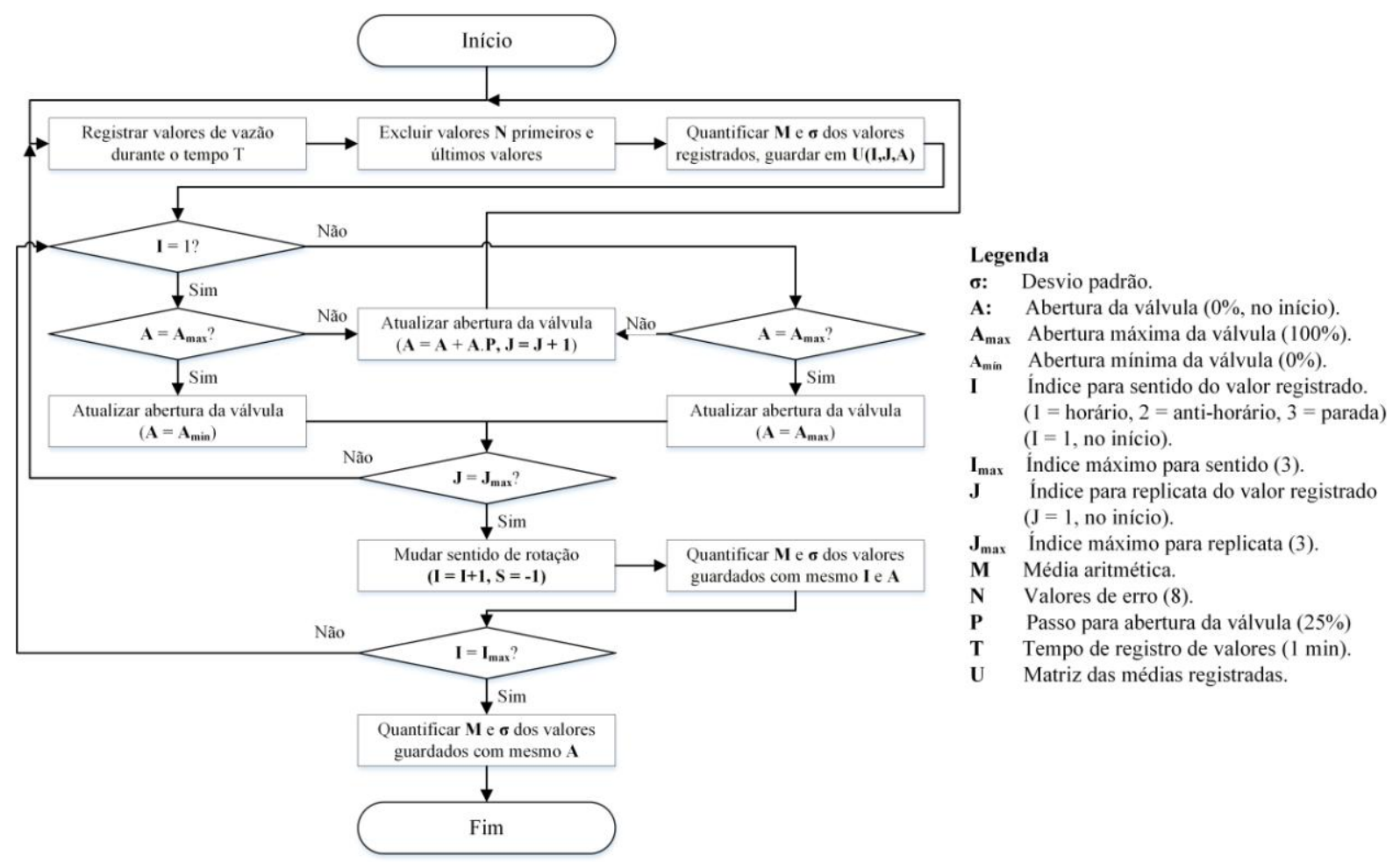

A figura 5 exibe a curva resultante da calibração da válvula, exibindo uma resposta do tipo raiz quadrada, apresentando um $\mathrm{R}^{2}$ de 0,9981 . Nela, observa-se o perfil quadrático da vazão em relação à abertura da válvula, o que é esperado pela válvula gaveta por possuir obstrução em formato de disco (SILVA, 2008). A tabela exibe os valores médios globais das vazões medidas e desvio padrão do experimento, o que atende a acurácia esperada para o medidor de vazão utilizado $(\geq 90 \%)$.

Figura 5 - Curva característica da válvula (instalada)

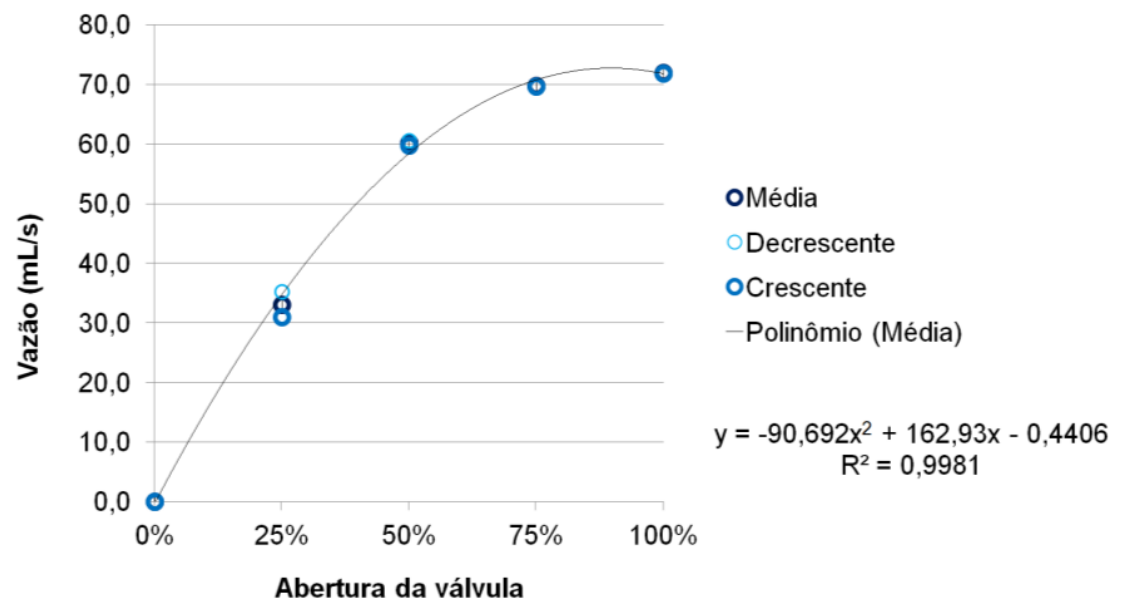

\begin{tabular}{|c|c|}
\hline $\begin{array}{c}\text { Abertura } \\
(\%)\end{array}$ & $\begin{array}{c}\text { Vazão } \\
(\mathrm{mL} / \mathrm{s})\end{array}$ \\
\hline 0 & $0,0 \pm 0,0$ \\
\hline 25 & $33,1 \pm 0,7$ \\
\hline 50 & $60,2 \pm 0,6$ \\
\hline 75 & $69,8 \pm 0,5$ \\
\hline 100 & $72,0 \pm 0,5$ \\
\hline
\end{tabular}

Abertura da válvula 


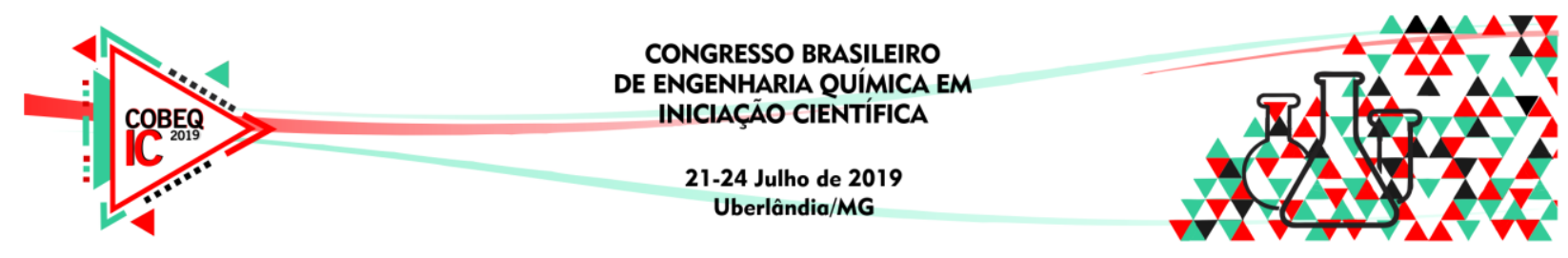

\section{CONCLUSÕES}

Uma planta piloto com recirculação de fluido promovida por uma bomba e um ponto de perda de vazão para ajuste à faixa de medição foi desenvolvida utilizando um balde cilíndrico, tubulação de PVC, válvula gaveta, bomba centrífuga, sensor de vazão e motor de passo. Com a construção da miniplanta, foi possível avaliar a relação entre a abertura da válvula e a vazão produzida, em meio a um fluxo de volume constante.

O circuito elétrico desenvolvido permite a utilização da instrumentação empregada na miniplanta. O algoritmo de controle desenvolvido viabiliza a comunicação entre os instrumentos e o sistema de acompanhamento, através dos mecanismos para comunicação OPC via servidor. Além disso, eles fornecem a energia necessária aos equipamentos, além de definir o ritmo para o funcionamento dos equipamentos, sendo assim de fundamental importância.

Os resultados alcançados por meio da curva de calibração permitiram caracterizar a válvula como do tipo raiz quadrada e o algoritmo desenvolvido para ajuste da abertura da válvula a partir do motor de passo permitem a aplicação da planta em controle de processos. A próxima etapa do projeto é fechar a malha de controle de vazão. A solução apresentada amplia a aplicabilidade da plataforma Arduino ao permitir a atuação contínua e automática da válvula de controle em processos químicos e bioquímicos.

\section{REFERÊNCIAS BIBLIOGRÁFICAS}

ANSI. ANSI/ISA 75.25.01-2000 (R.2010). Test Procedure For Control Valve Response Measurement From Step Inputs. United States of America: Instrumentation Systems and Automation Society, 2010.

BAUMANN, H. D. Control valve primer: a user's guide. $4^{\text {a }}$ edição. United States of America: Instrumentation Systems and Automation Society, 2009.

OSISOFT. PI System ${ }^{\mathrm{TM}}$ : From data to knowledge to transformation. United States of America: OSISOFT, 2019. Disponível em: <https://www.osisoft.com/pi-system/>. Acesso em 29 de março de 2019.

SILVA, O. J. L. da. Válvulas Industriais. Petróleo Brasileiro S.A. Rio de Janeiro: Qualitymark, 2008.

TELLES, P. C. S. Tubulações Industriais. 9ª edição. Rio de Janeiro: LTC, 2008.

ZHU, H.; ZHANG, J. Experiments Research for Field Calibration Method of HVAC Continuous Control Valves Characteristic. Proc. Eng. v. 205. p. 2141-2148, 2017. 